\title{
Alcune considerazioni a: Dialogo Aperto - un nuovo approccio psicoterapeutico e orientato ai diritti umani per costruire servizi psichiatrici umanistici
}

\author{
Giuseppe Riefolo*
}

Ho letto con interesse il contributo che Seikkula propone in questo numero. Premetto che non mi considero un esperto di Open Dialogue (OD), ma posso contare su una lunga esperienze di lavoro presso i servizi psichiatrici territoriali italiani simultaneamente ad un lavoro clinico come psicoanalista nella stanza di analisi. Premetto anche che per me le due posizioni sono profondamente connesse in quanto mi sento profondamente psicoanalista nella stanza di analisi che nei servizi. Peraltro, ho una lunga esperienza di interventi con pazienti psicotici e border in situazioni di acuzie o di trattamento ambulatoriale. In questa linea trovo profonda sintonia con l'approccio di OD. Concordo con la posizione centrale di OD è che il dialogo sia rappresentato non tanto nella sua funzione di 'comunicazione', quando nella dimensione di rappresentazione del soggetto e come atto creativo di significati nuovi' (p. 8). Inoltre, la comunicazione, nella linea di van der Kolk (2014), come per molti psicoanalisti soprattutto di impostazione intersoggettiva, ha la funzione di rendere narrabili e presentabili ad un interlocutore emozioni mai descritte prima. Ho pensato che, nella linea di alcune posizioni psicoanalitiche che suggeriscono la dissociazione come dispositivo fisiologico della organizzazione mentale (Bromberg, 2006), l'uso del dialogo possa essere rappresentato come un dispositivo che attiva il fisiologico 'processo dissociativo' rimuovendo la posizione di impasse del paziente caratterizzata, sul piano clinico, da blocco, ripetitività e rigidità (Ginot, 2015). Infine, non c'è da dubitare che 'un approccio basato sul bisogno' apra canali di condivisione e comunicazione con gli stati più bisognosi e feriti che sono alla base della sofferenza psicotica soprattutto acuta. Uno psicoanalista, ovviamente si chiede che cosa si intenda per 'bisogno' e, quindi, se nel cosiddetto ‘approccio basato sul bisogno' siano implicate - e in quale misu-

\footnotetext{
*Psichiatra, membro ordinario della Società Psicoanalitica Italiana.

E-mail: giusepperiefolo1@gmail.com
} 
ra - posizioni di collusione o suggestione (sappiamo elementi sempre presenti nei processi psicoterapeutici, ma il problema è come valutarli e che uso farne...). I risultati ovviamente documentano questo cambiamento importante rispetto alle TAU (terapie as usual). In questa linea viene documentato che, se ci si impegna nella cura (direi, in senso winnicottiano, nel prendersi cura) dei pazienti, magari sin dall'esordio, $i$ trattamenti risultano più economici per riduzione dei ricoveri, sussidi, pensionamenti e uso di farmaci.

A questo punto, e ribadendo un pieno riconoscimento delle potenzialità positive dell'approccio $\mathrm{OD}$, cercherò di discutere due quesiti. Da un lato sono interessato a capire quali siano i fattori specifici dell'approccio OD e dall'altro quali siano i riferimenti rispetto a cui $\mathrm{OD}$ si propone come più capace.

Riguardo al primo quesito, a mio parere la valutazione di un metodo terapeutico, ritengo non possa evitare una precisazione di che cosa intendiamo per 'fattori specifici e aspecifici'. Se ne sono occupati, necessariamente tutti colori, i quali cercano di valutare l'efficacia di un approccio terapeutico (Migone, 1996; Roth \& Fonagy, 1997; Stern 1995). Ebbene, la gran parte degli studi (io confesso che ne conosco solo alcuni e soprattutto quelli ad indirizzo psicoanalitico...) riconoscono che gli approcci terapeutici siano validi per i primi sei mesi indipendentemente dalle caratteristiche precise del modello utilizzato. I fattori aspecifici soprattutto comunicano al paziente la disponibilità all'accoglienza, il rispetto per la sintomatologia, la solidarietà per la sofferenza provata dal paziente, la condivisione per il dolore e l'impotenza insieme ad una sufficiente dose di speranza nella soluzione del blocco dovuto alla sofferenza psicotica. Ritengo importante, ma sostanzialmente di ordine aspecifico, che 'una volta che i sentimenti sono stati ampiamente condivisi nell'incontro, l'esperienza di guarigione relazionale diventa palpabile'. Gli elementi aspecifici ritengo siano la parte centrale e determinante del buon esito dell'approccio: l'intervento entro 24 ore; il coinvolgimento di tutti i soggetti significativi per la vita del paziente; il coinvolgimento diretto del paziente nella definizione del processo terapeutico, sono tutte posizioni di alta disponibilità soggettiva da parte dei terapeuti che sicuramente - indipendentemente dal modello clinico utilizzato - comporta necessariamente miglioramenti del quadro clinico. Ribadendo di non sentirmi un esperto conoscitore dell'OD, il quesito sulla specificità o meno dell'approccio OD rimane. Quelli che Seikkula riferisce come fattori specifici dell'approccio OD, possono essere tutti rivisti secondo altri modelli. Ad esempio: 'l'attenzione rivolta al rafforzamento del lato adulto del paziente e alla normalizzazione della situazione invece di concentrarsi sul comportamento regressivo', è un approccio anche della psicoanalisi relazionale, intersoggettiva e persino di orientamento ferencziano e winnicottiano. Semmai ci si potrebbe chiedere , in questo caso, che cosa si intenda per 'lato adulto del paziente'. L'approccio OD, evidentemente fa riferimento alla dimensione più operativa e di competenze concrete del paziente. L'approccio analitico relazionale si 
riferisce soprattutto alle competenze del paziente a sapersi riconoscere capacità dell'ordine creativo che, comunque, non sono in contraddizione con il riconoscimento di competenze concrete del paziente. Uno degli elementi aspecifici più importanti è la fiducia che il terapeuta cerca di guadagnarsi direttamente e concretamente - da parte del paziente. In questa linea, ovvio, anche, che i migliori risultati si ottengano quanto più il paziente o la situazione sia grave e che in questi casi la solidarietà sia una comunicazione di sintonizzazione potente che permette al paziente - come avrebbe potuto suggerire Kohut (1984) - che 'quando si empatizza con uno psicotico, da quel momento il paziente smette di essere tale'.

Dopo vengono i fattori specifici. Per un modello di terapia familiare ad orientamento cognitivo-comportamentale si tratta di sciogliere i vincoli patologici che legano i membri della famiglia e che fanno del paziente il portatore designato e più fragile dell'intero sistema patologico familiare. Per un approccio psicoanalitico si tratta di cogliere la comunicazione del paziente sul piano del transfert e della identificazione proiettiva restituendo al paziente le identificazioni modulate e trasformate dalla partecipazione dell'analista (Bion, Ogden, Bollas, Ferro...) o della sollecitazione agli acting-out dell'analista attraverso gli enactment indotti dal paziente (Bromberg, 2006; Boston Change Process Study Group, 2010; Boccara, Meterangelis, Riefolo, 2018) letti secondo codici di dialogo intersoggettivo e di reciprocità (Benjamin, 2017; Reis, 2020). Alla fine, i 'sette principi fondamentali' su cui si fonda l'OD potrebbero essere visti come sostanzialmente aspecifici e, quindi, comuni, ad altri approcci terapeutici, ovviamente di ordine dinamico. Ad esempio. Quando i membri dell'équipe si emozionano, sono chiamati a 'tollerare gli intensi stati emotivi indotti durante l'incontro'. Questo va bene, ma ci si può chiedere: che uso ne fanno poi i terapeuti della condivisione di stati affettivi intensi? Ovvio che il paziente, nella condivisione, si riconosca attivamente capace di modificare lo stato affettivo del caregiver (Stern, 1985), ma, quello stato emotivo in che modo e in quale misura appartiene al paziente (transfert) e, in quanto tale, pone il terapeuta in una dimensione di 'role responsiveness' (Sandler, 1976) o chiede al terapeuta una restituzione di elementi dissociati del proprio Sé che si sintonizzano con Stati dissociati del paziente?

Il secondo quesito che mi viene dalla lettura della proposta di Seikkula è di chiedersi a quali modelli si riferisca OD per ribadirne la specifica differenza e soprattutto le maggiori potenzialità terapeutiche. Un primo modello di confronto (direi inevitabile) è la psichiatria descrittiva e oggettivante. Infatti, l'OD si presenta come alternativo al 'discorso monologico rigido e ristretto'. Questo, ovviamente, permette positivi esiti, perché conosciamo bene la violenza e la scarsa disponibilità al dialogo da parte della psichiatria tradizionale. I riferimenti potrebbero essere di altro livello e, magari, più specifici. Ad esempio, quali possono essere i confronti con i ser- 
vizi per pazienti Border di cui si occupano Russel Meares in Australia (peraltro improntati proprio ad una dimensione di ampliamento creativo del dialogo...), oppure Marsha Linehan o Fonagy e Target con i loro modelli? Il confronto con la psichiatria tradizionale, a mio parere dice poco perché il livello della psichiatria tradizionale è notoriamente molto scarso.

Sul piano dinamico l'altro modello di confronto mi sembra sia la terapia familiare rappresentata nella sua dimensione di posizione codificata e chiusa in un rigido setting soprattutto quando si fa riferimento a 'professionisti abituati a lavorare in modo più strutturato' mentre l'approccio OD può permettere un ampliamento del setting ed una maggior sollecitazione alla partecipazione diretta del paziente. Concordo che soprattutto nei casi di gravi episodi o stati psicotici un setting di terapia familiare possa risultare di difficile applicazione e la proposta possa essere sentita distante e violenta da parte del paziente. In modo più implicito, mi sembra che un terzo modello di confronto sia quello psicoanalitico. Comunque, sempre nella linea della 'aspecificità' degli elementi strutturanti l'OD, ogni altro metodo terapeutico nelle situazioni psicotiche ritengo sia chiamato a modulare il proprio setting al fine di accogliere positivamente la sintomatologia psicotica del soggetto. Rispetto agli altri modelli, l'OD avrebbe il vantaggio di espandere tale posizione di 'accoglienza e solidarietà', attivamente all'intero contesto familiare del paziente e il coinvolgimento di una équipe ampia. È sicuramente un vantaggio, ma anche questo, ritengo dell'ordine aspecifico, che ha positivi esisti in quanto comunica la possibilità di sperimentare sintonizzazione relazionale che rompe la nicchia autistica in cui il paziente, difensivamente, si rifugia, nella esplosione della psicosi.

Un limite che riconosco è di almeno due ordini. Un primo limite è che mi sembra che l'OD si fermi alla 'condivisione e solidarietà' della sofferenza psicotica, senza coglierne la dimensione della comunicazione transferale che - secondo un registro psicotico - chiede al terapeuta di conoscere intimamente, potrei dire somaticamente (nel senso di un registro presimbolico, che magari si evidenzia attraverso l'azione, l'impotenza, il fallimento, gli errori e, quindi gli enactment) quale sia la natura specifica della sofferenza psicologica del paziente in quel preciso momento e con quel soggetto. Un altro limite che - sempre da psicoanalista - trovo nell'approccio dell'OD è una sorta di idealizzazione della 'buona pratica' rappresentata nella dimensione soprattutto concreta.

Per me il rischio è che si idealizzi una posizione teorica e clinica tenendola separata dalla realizzabilità concreta. In questo la mia posizione è precisa: come clinici siamo chiamati a 'fare il massimo che possiamo con quello che abbiamo' (Winnicott), mentre riferirsi a soluzioni ideali, ma concretamente irrealizzabili, rappresenta una posizione potenzialmente violenta di non assunzione della necessaria responsabilità clinica. Questa posizione negli anni ha costantemente portato a gravi posizioni di 'lamentazione' 
(Riefolo, 2017) da parte degli operatori verso la impossibilità frustrante a poter realizzare progetti sovradeterminati, mentre è nostro compito trovare sempre l'ambito che possa permettere il massimo livello di relazione (sintonizzazione) possibile col paziente anche quando questa sintonizzazione solleciti la condivisione di impotenza verso bisogni concreti. Un possibile dubbio potrebbe essere che l'approccio OD sia implicitamente organizzato sulle disponibilità concrete dei servizi di una società nord-europea e in particolare finlandese. Troverei molto violento che si proponesse un modello di intervento possibile in Finlandia non considerando le particolarità del contesto in cui noi siamo chiamati ad intervenire. Non è un problema di maggiore o minore disponibilità di risorse (infatti, conosciamo tutti come in alcuni casi vi sia un alto dispendio di risorse economiche con risultati anche anti terapeutici). Prima che di costi si tratta di un problema di cultura clinica dove un terapeuta sappia usare creativamente gli elementi concreti di cui - realmente - dispone (Riefolo, 2020). Sul piano dinamico, significa la possibilità di costruzione di setting specifici che sappiano accogliere la sofferenza dei pazienti e la fatica degli operatori permettendo processi di trasformazione della sofferenza psicotica. L'OD propone che si usino i 'setting naturali', ma questo è diverso - sul piano dinamico - dalla posizione di rendere setting gli elementi naturali disponibili che, prima che essere accettati, vanno usati. Il setting è un dispositivo specifico che attribuisce significato a ciò che accade. Un esempio semplice. Una cosa è accettare positivamente che il paziente chieda di pranzare da solo in soffitta durante la crisi psicotica finché non si sia rassicurato. Altra cosa è leggere i movimenti che il paziente fa rispetto al suo pranzare da solo o con gli altri come sua disponibilità a fidarsi dei terapeuti e quindi accedere alle cure. La differenza tra la prima e la seconda posizione è l'uso del setting secondo un registro naturale o transferale.

In psichiatria dinamica (soprattutto in psicoanalisi) il setting con le sue regole e i suoi 'limiti' è parte integrante del processo. Tutto ciò che accade (o non accade) nel setting ha immediatamente riferimento a dinamiche e potenzialità inconsce. Voglio dire che se in un servizio con gravi carenze concrete io faccio proposte concretamente irrealizzabili, questa non è semplicemente una 'svista', ma una posizione violenta e maniacale che risolve l'impotenza del paziente, non accogliendola e proiettandola violentemente su contesti che ci escludono (Riefolo, 2017). In sostanza bisogna prendere atto che l'OD in un contesto come quello italiano debba tener conto che non sia possibile, come nella descrizione del caso presentato, che 'medico di base possa contattare immediatamente uno psicologo presso l'ambulatorio di salute mentale locale' o che sia disponibile 'uno specifico luogo per crisi acute per garantire l'immediata organizzazione dell'incontro' e la possibilità che in un giorno si riesca ad organizzare una équipe di intervento sulla crisi e che questa équipe riesca ad effettuare 'una visita a casa lo stesso giorno nel pomeriggio' 
In sintesi: ribadendo la profonda convinzione che le tesi del OD siano estremamente interessanti e sicuramente valide sul piano psicodinamico, ho provato a discutere i possibili fattori specifici del metodo e, all'interno di una dinamica di setting, l'esito del processo di realizzabilità di queste posizioni essendo mia convinzione che la realizzabilità non sia separabile dal processo terapeutico e, particolarmente connessa con gli esiti delle cure.

\section{BIBLIOGRAFIA}

Benjamin, J. (2017). Il riconoscimento reciproco. Milano: Cortina.

Boccara, P., Meterangelis, G., Riefolo, G. (a cura di) (2018). Enactment. Parola e azione in psicoanalisi. Milano: Franco Angeli.

Boston Change Process Study Group (2010). Il cambiamento in psicoterapia. Milano: Cortina.

Bromberg, Ph. M. (2006). Destare il sognatore. Milano: Cortina.

Ginot, E. (2015). Neuropsicologia dell'inconscio. Milano: Cortina.

Kohut, H. (1984). La cura psicoanalitica. Torino: Bollati-Boringhieri.

Migone, P. (1996). La ricerca in psicoterapia: storia, principali gruppi di lavoro, stato attuale degli studi sul risultato e sul processo. Rivista Sperimentale di Freniatria, CXX(2), 182-238.

Reis, B. (2020). Il potere creativo. Dell'esperienza intersoggettiva. Roma: Astrolabio.

Riefolo, G. (2017). Dinamiche di lamentazione nei servizi territoriali. Rivista Sperimentale di Freniatria, CLI(1), 75-94.

Riefolo, G. (2020). Servizi. La parabola vitale del gruppo degli operatori. Rivista Sperimentale di Freniatria, CXLIV(3), 9-31.

Roth, A., Fonagy, P. (1997). Psicoterapie e prove di efficacia. Quale terapia per quale paziente. Roma: Il Pensiero Scientifico.

Sandler, J. (1976). Countertransference and role responsiveness. International Journal of Psychoanalysis, 3, 43-47.

Stern, D. (1985). Il mondo interpersonale del bambino. Torino: Bollati Boringhieri.

Stern, D. (1995). La costellazione materna. Torino: Bollati Boringhieri.

van der Kolk, B. (2014). Il corpo accusa il colpo. Milano: Cortina.

Conflitto di interessi: l'autore dichiara che non vi sono potenziali conflitti di interessi.

Approvazione etica e consenso a partecipare: non necessario.

Ricevuto per la pubblicazione: 9 agosto 2021.

Accettato per la pubblicazione: 28 agosto 2021 .

${ }^{C}$ Copyright: the Author(s), 2021

Licensee PAGEPress, Italy

Ricerca Psicoanalitica 2021; XXXII:589

doi:10.4081/rp.2021.589

This article is distributed under the terms of the Creative Commons Attribution

Noncommercial License (by-nc 4.0) which permits any noncommercial use, distribution,

and reproduction in any medium, provided the original author(s) and source are credited. 